\title{
An Estimator of Edge Length and Surface Area in Digitized 2D and 3D Images
}

\author{
Piet W. Verbeek and Lucas J. van Vliet \\ Pattern Recognition Group Delft, Faculty of Applied Physics, Delft University of Technology, \\ Lorentzweg 1, 2628 CJ Delft, The Netherlands
}

\begin{abstract}
Existing estimators for edge length in $2 D$ and surface area in $3 D$ are applied to a binary representation of the object. In this paper we estimate length and area through volume measurements. Volume is measured without thresholding and does not introduce a sampling error. Edges are transformed into volumes by giving them a constant height after which they can be shifted perpendicular to the edge over a small distance. Subtraction of two images shifted in opposite direction produces a volume that is proportional to the edge length. To guarantee a constant edge height along the edge or across the surface to be measured we apply a "soft" clipping operation to the linear region of the edge. For accurate and isotropic displacement of edges we introduce a continuous space equivalent to the discrete local minimum and maximum filters. These filters are sampling invariant and allow shifts in the subpixel region.
\end{abstract}

\section{Introduction}

The estimation of edge length such as the perimeter of an object from its digital image is a well-known problem. Practical recipes summarizing experimental results have been proposed [1] for the worst case in which only a binary image is available. Fitting an analytical curve to interpolated digital contour points and calculating its length is a more theoretically founded approach [2].

We believe that in the thresholding that usually produces the binary image valuable information is lost that should be preserved to improve the estimation of edge length.

The method presented here works on grey value images and combines edge detection and edge length estimation. Apart from a few plausible assumptions it is founded on sampling theory.

\section{Edge length estimation in the discrete domain is a problem, volume estimation is not}

Edge detection is generally based on thresholding. The simplest example is thresholding of the original grey image at a fixed level, e.g. half edge height. Thresholding the original image at a space variant level (a reference image that can be derived from the original by low-pass filtering or local minimum and maximum filtering [4]) is equivalent to high pass filtering and thresholding at a fixed level. In particular, one often thresholds a second derivative at level zero. This introduces noise sensitivity but solves the level selection problem.

Thresholding is a very nonlinear operation in the sense that the Taylor expansion of the corresponding scaling function $\mathrm{T}$ (grey level) $\rightarrow\{0,1\}$ has appreciable higher-order terms. Consequently, [3] thresholding in the continuous domain can only be replaced by an equivalent digital operation "sampling $\rightarrow$ digital thresholding $\rightarrow$ interpolation" at the cost of equally appreciable oversampling.

By contrast, the volume of a grey value landscape (integrated grey value) is equal to the sum of the samples if only the Nyquist criterion is fulfilled (no oversampling). This has led us to look for an estimation method where edge length is expressed in a volume measure.

\section{Constant height edges}

\subsection{Straight edges}

To start with, we employ our argument in the continuous domain. In order to convert an edge length into a volume of a grey value landscape proportional to it we can see from dimension considerations that we must multiply by a constant width and a constant height. For an edge of constant grey level (constant

P.W. Verbeek and L.J. van Vliet, An estimator of edge length and surface area in digitized 2D and 3D images, in: proc. $11^{\text {th }}$ IAPR International Conference on Pattern Recognition, The Hague (The Netherlands), vol. III, 1992, 1-5. 
edge range) this value could be used as constant height. But the problem remains how to introduce a constant width.

The solution we found is to apply an analog round local maximum filter of diameter size to the analog edge. The effect of which is mainly a sideways translation of the edge over the filter radius.

The volume enclosed between the original and the translated edge is then equal to

volumeof $($ max - ori $)=$ length $_{\text {edge }} \cdot \frac{1}{2}$ size $\cdot$ edge range

with $\max$ being the result after maximum filtering and ori the original edge image.

For a straight edge the relation is exact as long as the maximum filtering truly amounts to translation. We shall discuss ways to ensure this.

\subsection{Curved edges}

For a curved edge, maximum filtering will not only translate the edge, but will also change the edge radius. From the simple example of a circular step edge we see that it is then better to apply two filters, an analog round local maximum filter and an analog round local minimum filter, to the original edge. The volume enclosed between the results is then equal to

$$
\operatorname{volumeof}(\max -\min )=\text { length }_{\text {edge }} \cdot \text { size } \cdot \text { edge }_{\text {range }}
$$

For an edge of different shape it is more complicated to define edge position and edge length. There are several traditional definitions of edge position. The simplest is based on thresholding which produces a binary edge. In section 6 we show how edge length according to other traditional definitions such as the zero crossing of a second derivative - can be handled. For now, we remark that eq. (2) holds for one particular uncommon definition of edge position (cf. fig.1): the average (continuous) position $r_{\text {eff }}$ of the binary edge in a cross section perpendicular to the edge, when the average is taken over the entire interval of threshold levels (0, edge height).
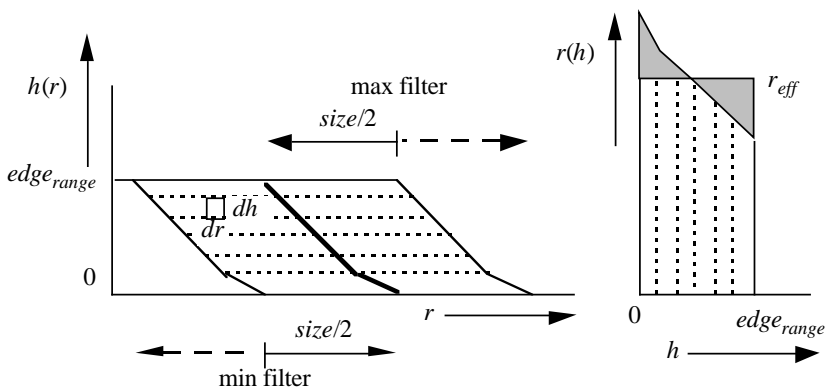

Figure 1: Cross section perpendicular to an edge of constant height.
This follows immediately from the expression below for a sector $d \phi$ of the enclosed volume

$$
\begin{aligned}
& \frac{\partial \text { volumeof }(\max -\min )}{\partial \varphi}=\int_{0}^{e d g e_{\text {height }}} \int_{r(h)-\frac{1}{2} \text { size }}^{r(h)+\frac{1}{2} \text { size }} r d r d h \\
& =\frac{1}{2} \int_{0}^{e d g e_{\text {range }}}\left[\left(r(h)+\frac{\text { size }}{2}\right)^{2}-\left(r(h)-\frac{\text { size }}{2}\right)^{2}\right] d h \\
& =\operatorname{size} \int_{0}^{e d g e_{\text {range }}} r(h) d h \equiv \text { size } \cdot e d g e_{\text {range }} \cdot r_{\text {eff }}
\end{aligned}
$$

\section{Isophote edge length}

In practice the edge height will show ripple and noise. To achieve a simulated constant height we apply clipping at two levels clip $_{\text {low }}$ and cliphigh $_{\text {his }}$. This amounts to using a more general definition of edge position (cf. fig.2): the average (continuous) position $r_{\text {eff }}$ of the binary edge in a cross section perpendicular to the edge, when the average is taken over an interval of threshold levels $\left(\right.$ clip $_{\text {low }}$, clip $\left._{\text {high }}\right)$.
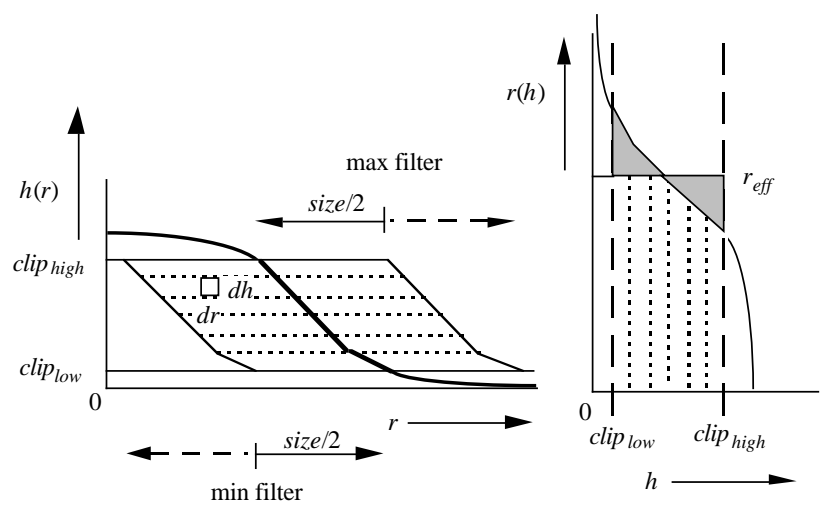

Figure.2: Cross section perpendicular to the edge; $r_{\text {eff }}$ is the average position of the binary edge, when the average is taken over an interval of threshold levels (cliplow, cliphigh).

In particular, the clipping levels can be chosen close together around a fixed "threshold" level $t$. Then the length calculated is that of the isophote at level $t$.. As we are still handling continuous positions we have constructed a continuous analogy of contour length. Just like discrete contour length, our edge length depends on a "threshold" level.

\section{Continuous edge length from a sampled image}

When assessing the volume that is representative for edge length it makes little difference whether the image is sampled or not. For a bandlimited image the 
integral over continuous grey value equals the sum of the grey value samples.

Two operations used to convert edge length into volume need attention when assessing continuous contour length from a sampled image:

- the continuous maximum filter used for translation of the edge must be replaced by a discrete operation which on the basis of the sampled image yields the sample values of the translated continuous edge,

- the clipping operation being a nonlinear scaling function must be handled with care.

In both issues an approximation is proposed to make a practical compromise between bandlimitation and accuracy.

Edge translation over size/2 is approximated by a truncated Taylor expansion.

$$
h\left(r+\frac{\text { size }}{2}\right)=h(r)+\frac{\text { size }}{2} \frac{\partial h(r)}{\partial r}+\frac{1}{2}\left(\frac{\text { size }}{2}\right)^{2} \frac{\partial^{2} h(r)}{\partial r^{2}}
$$

In terms of the 2D image this reads ( $r$ is along the gradient direction)

$$
\begin{gathered}
h\left(r+\frac{\text { size }}{2}\right)=h(x, y)+\frac{\text { size }}{2}|\operatorname{grad}(h(x, y))| \\
+\frac{1}{2}\left(\frac{\text { size }}{2}\right)^{2} \operatorname{SDGD}(h(x, y))
\end{gathered}
$$

where

$$
|\operatorname{grad}(h(x, y))|=|\operatorname{grad}(h)|=\sqrt{\left(\frac{\partial h}{\partial x}\right)^{2}+\left(\frac{\partial h}{\partial y}\right)^{2}}
$$

and SDGD stands for Second Derivative in the Gradient Direction and can be written as

$$
\operatorname{SDGD}(h)=\frac{\frac{\partial^{2} h}{\partial x^{2}}\left(\frac{\partial h}{\partial x}\right)^{2}+2 \frac{\partial^{2} h}{\partial x y} \frac{\partial h}{\partial x} \frac{\partial h}{\partial y}+\frac{\partial^{2} h}{\partial y^{2}}\left(\frac{\partial h}{\partial y}\right)^{2}}{\left(\frac{\partial h}{\partial x}\right)^{2}+\left(\frac{\partial h}{\partial y}\right)^{2}}
$$

Although the derivatives are all bandlimited, the modulus of the gradient and the SDGD convert a bandlimited image into results that can only be hoped to be approximately bandlimited. The reason for this hope is in the fact that non-linearity of the modulus and of the SDGD does hardly occur in the center part of the edges which we select for edge length estimation.

Clipping a sampled image is tantamount to clipping the continuous image and sampling; clipping the continuous image distorts the grey value landscape so that it is no longer bandlimited. (The distortion is already much weaker than the one caused by thresholding.) In order to reduce aliasing we propose to replace the grey scaling function of clipping by a smoother function, the error function, between the same levels.

$$
\begin{aligned}
& \operatorname{clip}_{\text {erf }}(h(r))=\operatorname{clip}_{\text {low }} \\
& +\operatorname{clip}_{\text {range }} \operatorname{erf}\left(\frac{h(r)-\frac{1}{2}\left(\text { clip }_{\text {low }}+\text { clip }_{\text {high }}\right)}{\frac{2}{\sqrt{\pi}} \text { clip }_{\text {range }}}\right)
\end{aligned}
$$

with clip $_{\text {range }}=\left(\right.$ clip $_{\text {high }}-$ clip $\left._{\text {low }}\right)$.

In cross sections where the edge slope between clipping levels is approximately constant

$$
h(r)=a r+b
$$

the edge is shaped into a scaled error function

$$
\begin{aligned}
& \operatorname{clip}_{\text {erf }}(h(r))=\text { clip }_{\text {low }} \\
& +c_{\text {range }} \operatorname{erf}\left(\frac{a r+b-\frac{1}{2}\left(\text { clip }_{\text {low }}+\text { clip }_{\text {high }}\right)}{\frac{2}{\sqrt{\pi}} \text { clip }_{\text {range }}}\right)
\end{aligned}
$$

the approximate bandwidth of which is

$$
f_{\max e r f}=\frac{a \sqrt{2} / \pi}{2\left(\text { clip }_{\text {high }}-\text { clip }_{\text {low }}\right) / \sqrt{\pi}}
$$

(lemma 1) limited by (lemma 2)

$$
f_{\text {max erf }}=\frac{a}{\sqrt{2 \pi} \text { clip }_{\text {range }}} \leq \frac{1}{2} \sqrt{2 \pi} \frac{f_{\text {max }} \text { grey }_{\text {range }}}{\text { clip }_{\text {range }}}
$$

Hence, if the clipping interval and the linear part of the edge slope encompasses $1 / k$ of the total grey range, the erf clipping needs $k$ times oversampling $\left(\frac{1}{2} \sqrt{2 \pi} \approx 1\right)$. The value $k=4$ seems reasonable and practical.

lemma 1

As a signal and its derivative have the same bandwidth, we take for the approximate bandwidth of the error function the value of $2 \sigma_{\text {freq }}=2 /(2 \pi \sigma)$ of the gauss function of which it is the integral. As the error function is defined as $\operatorname{erf}(z) \equiv \frac{2}{\sqrt{\pi}} \int_{0}^{z} \exp \left(-t^{2}\right) d t$, $\operatorname{erf}(c r)=\frac{2}{\sqrt{\pi}} \int_{0}^{r} \exp \left(-c^{2} t^{2}\right) d t$, based on a Gaussian with variance $\sigma^{2}=\frac{1}{2} c^{-2}$. Thus, $\sigma_{\text {freq }}=1 /(2 \pi \sigma)=c /(\pi \sqrt{2})$ and the approximate bandwidth $=2 \sigma_{\text {freq }}=(c \sqrt{2}) / \pi$. 


\section{lemma 2}

The original slope is limited by $a \leq a_{\max }$ $\equiv 2 \pi f_{\max } \frac{1}{2}$ grey $_{\text {range }}$ where $f_{\max }$ is the maximum frequency in the original cross section.

\section{proof of lemma 2}

The steepest slope occurs if all allowed frequencies add up with the same phase

$$
\left.\frac{\partial}{\partial t} \int A(f) \sin (2 \pi f t) d f\right|_{t=0}=2 \pi \int A(f) f d f
$$

the maximum signal value that can occur is

$$
\left.\int A(f) \cos (2 \pi f t) d f\right|_{t=0}=\int A(f) d f
$$

The spectrum that gives the steepest slope with respect to the maximum signal $h_{\max }=\frac{1}{2}$ grey $_{\text {range }}$ has amplitude $A(f)=h_{\max } \delta\left(f-f_{\max }\right)$, with steepest slope

$$
a_{\max }=2 \pi f_{\max } h_{\max }=2 \pi f_{\max } \frac{1}{2} \text { grey }_{\text {range }}
$$

\section{Zerocrossing of second-derivative edge length}

When edge height varies slowly like in shading, the length of an isophote does not represent edge length properly. The edge is then defined as the position where a weighted sum of second derivatives along and perpendicular to the edge crosses zero. We shall loosely call this "the second derivative" (secder). Examples are: the Laplacian, the second derivative in the gradient direction and their sum $[5,6]$. We now define the local "threshold" $t_{l o c}$ in such a way that it intersects the original edge where the second derivative crosses zero. The easiest way to achieve this is to take

$$
t_{l o c}=\text { ori }+\alpha \text { secder }
$$

with $\alpha$ chosen so as to yield a constant $t_{l o c}$ in the cross section perpendicular to the edge, i.e.

$$
\begin{aligned}
& \operatorname{grad}\left(t_{\text {loc }}\right) \bullet \operatorname{grad}(\text { ori })=0 \\
& \alpha^{-1} \approx-\operatorname{grad}(\text { ori }) \bullet \operatorname{grad}(\text { secder }) / \operatorname{grad}^{2}(\text { ori })
\end{aligned}
$$

\section{Experiments and conclusions}

The test images contain a simulated image of a step edge imaged through an optical system and sampled at the Nyquist frequency $(1 \mathrm{~N})$ and at four times the Nyquist frequency (4N). Such an edge is constructed under various orientations with respect to the square sampling grid in order to detect directional dependencies. Bandlimited disk images are constructed in a similar way. To construct an arbitrary bandlimited object, we start out from its Fourier transform, ensure proper bandlimitation by multiplying with the OTF (optical transfer function), and apply an inverse Fourier transform to obtain the desired image. From eq. (4) we notice that the SDGD is used to shift the edge. The operator $\operatorname{SDGD}($ ori $) \mid\left.\operatorname{grad}($ ori $)\right|^{2}$ requires three times oversampling [3]. In order to avoid spurious aliasing effects by shifting the edge, the image must at least be sampled at three times the Nyquist frequency (even then it may introduce some aliasing because in the shift procedure we use $\operatorname{SDGD}($ ori), dividing $\operatorname{SDGD}($ ori $) \mid\left.\operatorname{grad}($ ori $)\right|^{2}$ by $\mid\left.\operatorname{grad}($ ori $)\right|^{2}$ is not guaranteed to be bandlimited). If a fixed optical system and image sensor cannot provide this, then the bandwidth must be reduced by a digital low-pass filter such as a Gaussian filter. Earlier work [6] showed that (applied to a Nyquist sampled signal) a Gaussian filter with $\sigma=2.7$ reduces the bandwidth by a factor of three, as needed.

The digital implementation can be achieved using two different strategies.

In the first strategy, we start with clipping around a certain "threshold" to ensure a constant edge height. The result of clipping is then shifted perpendicularly to the edge according to eq. (4). Following eq. (2), the edge length is given by

$$
\text { length }_{\text {edge }}=\text { volumeof }\left(\mid \operatorname{grad}(\text { ori }) \mid / \text { clip }_{\text {range }}\right)
$$

We notice that the SDGD does not contribute to the final result.

The second strategy starts with shifting the edge image, selecting a suitable "threshold" level and apply the clipping around the selected "threshold". Now the measured edge length is given by

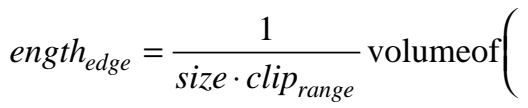

$$
\begin{aligned}
& \operatorname{clip}_{\operatorname{erf}}\left(\text { ori }+\frac{\text { size }}{2} \mid \operatorname{grad}(\text { ori }) \mid+\frac{\text { size }^{2}}{8} \operatorname{SDGD}(\text { ori })\right) \\
& \left.-\operatorname{clip}_{\mathrm{erf}}\left(\text { ori }-\frac{\text { size }}{2} \mid \operatorname{grad}(\text { ori }) \mid+\frac{\text { size }^{2}}{8} \operatorname{SDGD}(\text { ori })\right)\right)
\end{aligned}
$$

The filters used to build $|\mathrm{grad}|$ and SDGD are the first and second derivatives of a Gaussian. In order to guarantee isotropy, all partial derivatives used to construct the SDGD must have the same built-in low-pass filter, equal $\sigma$. At the same time this $\sigma$ is used to avoid spurious aliasing.

For curved edges the Gaussian filter built in the derivative filters virtually shifts the isophote inwards. The reason is that for a filter with center on the 
isophote (starting from $50 \%$ overlap for a straight edge) the overlap between filter and object will decrease with increasing curvature. This will happen, even if (minimizing edge displacement by OTF and noise combatting low pass filters) we base the isophote on the PLUS edge detector.

The first strategy allows us to choose the following parameters:

- the threshold level and clipping range,

- the built-in $\sigma$ of the derivative filters.

The second strategy has one additional parameter:

- the size of the analog max and min filters

For strategy 1, the experiments show that the type of clipping (soft clipping using the error function or hard clipping) does not make any difference here. Sampling at the Nyquist frequency seems to be sufficient (surprisingly, oversampling is not needed), since all measurements with small built-in $\sigma$ worked well (cf. table 1). For straight edges, the edge length was independent of the "threshold" level and clipping range. For circular edges the isophotes have different length. A larger $\sigma$ reduces the length of the isophote at half height and produces a systematic underestimate of the contour length. Since the edge radius is not a priori known we have chosen not to correct for this error [5].

Table 1: Relative errors in the measured edge length using strategy 1

\begin{tabular}{|c|c|c|c|c|}
\hline bandlimited edges & $\sigma=1.0$ & $\sigma=1.5$ & $\sigma=2.0$ & $\sigma=2.5$ \\
\hline Straight & $0.5 \%$ & $0.3 \%$ & $.1 \%$ & $0.0 \%$ \\
\hline $0^{\circ}-45^{\circ}$ & $0.2 \%$ & $0.1 \%$ & $0.1 \%$ & $0.0 \%$ \\
\hline straight edge $0^{\circ}$ & $0.0 \%$ & $0.0 \%$ & $0.0 \%$ & $0.0 \%$ \\
\hline straight edge $5^{\circ}$ & $0.5 \%$ & $0.3 \%$ & $0.1 \%$ & $0.0 \%$ \\
\hline straight edge $22.5^{\circ}$ & $0.0 \%$ & $0.0 \%$ & $0.0 \%$ & $0.0 \%$ \\
\hline straight edge $45^{\circ}$ & $0.0 \%$ & $0.0 \%$ & $0.0 \%$ & $0.0 \%$ \\
\hline circular edge & $<0.4 \%$ & $<0.5 \%$ & $<0.8 \%$ & $<1.2 \%$ \\
\hline $15<\mathrm{R} \leq 32$ & $0.2 \%$ & $0.3 \%$ & $0.5 \%$ & $0.7 \%$ \\
\hline circular edge & $<0.3 \%$ & $<0.2 \%$ & $<0.3 \%$ & $<0.4 \%$ \\
\hline $33 \leq \mathrm{R} \leq 50$ & $0.2 \%$ & $0.1 \%$ & $0.2 \%$ & $0.2 \%$ \\
\hline circular edge $R=15$ & $0.2 \%$ & $-0.3 \%$ & $-0.7 \%$ & $-1.2 \%$ \\
\hline circular edge $R=32$ & $0.1 \%$ & $-0.1 \%$ & $-0.3 \%$ & $-0.4 \%$ \\
\hline circular edge $R=50$ & $0.2 \%$ & $-0.0 \%$ & $-0.1 \%$ & $-0.1 \%$ \\
\hline
\end{tabular}

For strategy 2, the experiments show that only soft clipping gives a good length measure. Hard clipping causes unacceptable errors (at best $10 \%$ and much too sensitive to the choice of $\sigma$ ) for straight edges under $0^{\circ}$ and $45^{\circ}$. For soft clipping the straight edge under $0^{\circ}$ turned out to be the most sensitive to parameter choices $(\sigma$, and size). An acceptable sensitivity could not be achieved when omitting the SDGD contribution to the edge shift. Typical combinations are shown in table 2 and 3.

Table 2: Relative errors in the measured edge length using strategy 2 for straight edges

\begin{tabular}{|lr|r|r|}
\hline bandlimited edges & $\begin{array}{r}\sigma \geq 4 \\
\end{array}$ & $\begin{array}{r}\sigma \geq 1 \\
\text { size/2=0.3 } \\
1 \times \text { xyquist }\end{array}$ & $\begin{array}{r}\text { size/2 }=0.3 \\
4 \mathrm{x} \text { Nyquist }\end{array}$ \\
\hline straight edge & $0.2 \%$ & $0.1 \%$ \\
$0^{\circ}-45^{\circ}$ & $0.1 \%$ & $0.0 \%$ \\
\hline
\end{tabular}

Table 3: Relative errors in the measured edge length using strategy 2 for circular edges

\begin{tabular}{|lr|r|r|r|}
\hline bandlimited edges & $\begin{array}{r}\sigma=2 \\
\text { size/2=0.3 } \\
\text { 1xNyquist }\end{array}$ & $\begin{array}{r}1 \leq \sigma \leq 2.5 \\
\text { size/2=0.3 } \\
\text { 4xNyquist }\end{array}$ & $\begin{array}{r}\sigma \geq 1 \\
\text { size } / 2=0.3 \\
\text { 8xNyquist }\end{array}$ \\
\hline circular edge & max & $0.5 \%$ & & \\
$15 \leq \mathrm{R} \leq 30$ & mean & $0.3 \%$ & & \\
circular edge & max & & $0.2 \%$ & \\
$40 \leq \mathrm{R} \leq 121$ mean & & $0.1 \%$ & \\
circular edge & max & & & $0.1 \%$ \\
$80 \leq \mathrm{R} \leq 140$ mean & & & $0.0 \%$ \\
\hline
\end{tabular}

A 3D implementation to measure surface area is under way.

\section{Acknowledgement}

This work was partially supported by the Dutch Government as part of the SPIN-FLAIR II program "Delft Intelligent Assembly Cell", the SPIN-3D program for 3D biomedical image analysis and by The Netherlands Foundation for Biomedical Research NWO-MEDIGON, grant 900-538-016.

\section{References}

[1] Young, I.T., Sampling density and quantitative microscopy, Analytical and Quantitative Cytology and Histology, vol. 10, no. 4, 1988, pp. 269-275.

[2] Mullikin, J.C., Boundary description and measurement with sub-pixel/-voxel accuracy, submitted to ICPR 1992, 1991.

[3] Verbeek, P.W., A class of sampling-error free measures in oversampled bandlimited images, Pattern Recognition Letters, vol. 3, 1985, pp. 287-292.

[4] Verbeek, P.W., H.A. Vrooman and L.J. van Vliet, Low level image processing by max-min filters, Signal Processing, vol. 15, no. 3, 1988, pp. 249-258.

[5] Verbeek, P.W., and L.J. van Vliet, On the location error of curved edges in low-pass filtered 2D and 3D images I: Theory, submitted to IEEE PAMI, 1991.

[6] Van Vliet, L.J. and P.W. Verbeek, On the location error of curved edges in low-pass filtered 2D and 3D images II: Application Aspect, submitted to IEEE PAMI, 1991. 